\title{
Accuracy of the Low-Dose ACTH Stimulation Test for Adrenal Insufficiency Diagnosis: A Re-Assessment of the Cut-Off Value
}

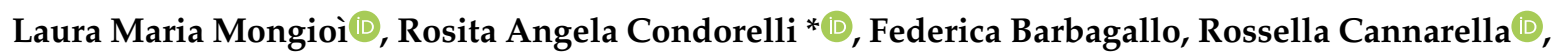 \\ Sandro La Vignera $₫$ and Aldo Eugenio Calogero \\ Department of Clinical and Experimental Medicine, University of Catania, Via S. Sofia 78, 95123 Catania, Italy; \\ lauramongioi@hotmail.it (L.M.M.); federica.barbagallo11@gmail.com (F.B.); roxcannarella@gmail.com (R.C.); \\ sandrolavignera@unict.it (S.L.V.); acaloger@unict.it (A.E.C.) \\ * Correspondence: rosita.condorelli@unict.it; Tel.: +39-95-3782311
}

Received: 13 April 2019; Accepted: 1 June 2019; Published: 5 June 2019

\begin{abstract}
Background: The clinical practice shows that many low-dose ACTH-stimulation tests have a false positive result. The aim of the study was to determine the diagnostic accuracy of a low-dose ACTH-stimulation test in the diagnosis of adrenal insufficiency and to define its optimal cut-off. Methods: We analyzed data from 103 patients undergoing $1 \mu \mathrm{g}$ ACTH-stimulation test. Four patients had adrenal insufficiency (AI) upon follow up: Two primary, and two secondary AI. Cortisol serum levels were evaluated at time $0,20^{\prime}$, and 30' after the injection of $1 \mu \mathrm{g}$ i.v. of ACTH. The sensitivity, specificity, accuracy, and positive and negative predictive values of the test were calculated for both $20^{\prime}$ and 30' sampling. The receiver operating characteristic (ROC) curve was obtained to assess the sensitivity and specificity of low-dose ACTH-stimulation test in the diagnosis of adrenal insufficiency at different cut-off values. Results: Considering $500 \mathrm{nmol} / \mathrm{L}$ as the standard cut-off value, low-dose ACTH stimulation test showed a $100 \%$ sensitivity and a $67.3 \%$ specificity, with a high rate of false positive results. ROC curve analysis showed that the cut-off of $401.5 \mathrm{nmol} / \mathrm{L}$ is the best compromise between sensitivity (100\%) and specificity (93.9\%). Conclusions: By using a cut-off value of 401.5 $\mathrm{nmol} / \mathrm{L}$ for the low-dose ACTH stimulation test, the number of false positive patients decreased significantly, but the sensitivity remained high.
\end{abstract}

Keywords: adrenal insufficiency; cortisol levels; low-dose ACTH test stimulation

\section{Introduction}

Adrenal insufficiency (AI) is a rare, life-threatening disease caused by either primary adrenal failure [1] or by hypothalamic-pituitary impairment of the corticotropic axis alone or in combination with other anteropituitary hormone deficiencies (CPHD) [2]. AI, even to a mild degree, is associated with increased mortality; therefore, a timely diagnosis is essential [3].

The diagnosis of $\mathrm{AI}$ is often difficult due to an onset of non-specific symptoms over the period of time (sometimes many years). In past years, the most validated test for the integrity of the hypothalamic-pituitary-adrenal (HPA) axis was the cortisol response to insulin-induced hypoglicaemia, known as the insulin stress test (IST). However, this test is largely considered too dangerous to perform. According to the Endocrine Society, the corticotropin (ACTH) stimulation test is the new diagnostic "gold standard" for the diagnosis of AI, although there is still some debate about the cut-off value of cortisol after stimulation. Moreover, different protocols are used in clinical practice with respect to the duration of the test, intramuscular or intravenous drug administration, and dose of corticotropin administered [4]. Usually, the standard short ACTH test measures cortisol levels at 30 and 60 minutes 
after the administration of $250 \mu \mathrm{g}$ of corticotropin as an intravenous bolus. Traditionally, peak cortisol levels below $500 \mathrm{nmol} / \mathrm{L}(18 \mu \mathrm{g} / \mathrm{dL})$ are suggestive of AI, but the results are assay-dependent [4]. In clinical practice, low-dose ( $1 \mu \mathrm{g}$ of corticotrophin) ACTH stimulation testing is often used, since it gives results comparable or even more sensitive than that of high-dose $(250 \mu \mathrm{g})$ testing [4-6].

The aim of this study was to evaluate if a cut-off different from $500 \mathrm{nmol} / \mathrm{L}$ for $1 \mu \mathrm{g}$ ACTH stimulation test could increase its sensitivity and specificity in the diagnosis of AI.

\section{Material and Methods}

The test was performed in 103 consecutive patients (20 males and 83 females, mean age: $39.6 \pm 1.47$ years) attending the Division of Andrology and Endocrinology, Teaching Hospital "Policlinico-Vittorio Emanuele," University of Catania between July 2016 and July 2018, with signs and/or symptoms suggestive of $\mathrm{AI}$ and cortisol levels between $82.75 \mathrm{nmol} / \mathrm{L}$ and $500 \mathrm{nmol} / \mathrm{L}$. As the symptoms are similar in both primary and central forms, it is not always possible to distinguish between the two forms. Although ACTH levels are useful for this purpose, in some cases they do not allow this objective to be achieved. In this study, before testing, $49 \%(n=50)$ of patients were suspected to have a primary form of AI, since their basal cortisol levels were $<276 \mathrm{nmol} / \mathrm{L}$, and ACTH levels were normal or high, and many of them had chronic autoimmune thyroiditis and/or other autoimmune diseases. Instead, $17 \%(n=18)$ of patients were suspected to have a central form of AI, as they presented hypothalamic-pituitary diseases (mainly pituitary adenoma) currently or in the past years. Finally, for $33 \%(n=34)$ of patients it was not possible to hypothesize the etiology of the possible $\mathrm{AI}$ (cortisol levels $<276 \mathrm{nmol} / \mathrm{L}$ and ACTH normal levels in absence of other suggestive data).

Each patient who participated in this study was asked to sign a written informed consent form and the Intra-Divisional Ethic Committee of the Endocrinology Section approved the research protocol.

We excluded from this study patients who had used glucocorticoids in the last two weeks before the test was performed. The presence of severe comorbidities was also considered the exclusion criterion.

Cortisol serum levels were evaluated at zero, 20, and 30 minutes after the intravenous injection of $1 \mu \mathrm{g}$ of 1-24 tetracosactid ( $1 \mu \mathrm{g}$ ACTH stimulation testing). Every test was performed at 8.00 AM. Serum cortisol concentrations were determined by an enzyme-linked immunocolorimetric assay in an automated procedure.

Sensitivity, specificity, positive and negative predictive values, and accuracy of the test were calculated. The receiver operating characteristic (ROC) curve was obtained to assess the sensitivity and specificity of $1 \mu \mathrm{g}$ ACTH stimulation testing in the diagnosis of AI at different cut-off points.

\subsection{Statistical Analysis}

Sensitivity, specificity, positive (PPV) and negative (NPV) predictive values, and accuracy were calculated using the number of patients' true-positive (TP), true-negative (TN), false-positive (FP) and false-negative (FN) results. PPV (calculated as TP / (TP + FP) was defined as the likelihood that a subject with a positive test (peak cortisol below the specific cut-off point) was affected with primary AI. NPV (calculated as TN / (TN + FN) was defined as the likelihood that a subject with a negative test (peak cortisol above the specific cut-off point) has a normal adrenal function. By repeated-measures analysis of variance (ANOVA) with a Greenhouse-Geisser correction, we compared the serum cortisol levels of each patient at 0,20 , and 30 minutes. The receiver operating characteristic (ROC) curve was obtained to assess the sensitivity and specificity of the $1 \mu \mathrm{g}$ ACTH stimulation test in the diagnosis of $\mathrm{AI}$ at different cut-off values for 20- and 30-minute serum cortisol levels during the test. The area under the ROC curve (AUC) was also calculated. The ROC curve at 30 minutes was used to determine the optimal cut-off value. McNemar's test was used to compare the number of FPs between the standard cut-off of $500 \mathrm{nmol} / \mathrm{L}$ and the optimal cut-off value determined through the ROC curve.

A statistical analysis of the data was performed using the Statistical Package for the Social Sciences version (SPSS) 23.0 for Windows software program (IBM Corp., Armonk, NY, USA). Results with a $p$ value of less than 0.05 were considered to be statistically significant. 


\section{Results}

One female patient dropped out the study, since she did not initially report that she took glucocorticoids the day before the test. Table 1 summarizes the principal features of the whole population studied and of patients with AI.

Table 1. Demographic characteristics of the cohort of patients included in the study and in those with AI.

\begin{tabular}{ccc}
\hline Parameters & All Patients & AI \\
\hline Female & 82 & 3 \\
\hline Male & 21 & 1 \\
\hline Age & $39.6 \pm 14.5$ & $40.3 \pm 14.2$ \\
\hline Weight & $68.8 \pm 15.5$ & $78.4 \pm 7.4$ \\
\hline BMI & $26.7 \pm 5.5$ & $30.9 \pm 3$ \\
\hline & AI $=$ Adrenal insufficiency.
\end{tabular}

Of the remaining 102 patients, 66 patients showed a normal response to ACTH stimulation, with peak cortisol levels greater than $500 \mathrm{nmol} / \mathrm{L}(18 \mu \mathrm{g} / \mathrm{dL})$. Among these, seven patients showed a peak cortisol level of greater than $500 \mathrm{nmol} / \mathrm{L}$ at 20 minutes and a value lower than $500 \mathrm{nmol} / \mathrm{L}$ at 30 minutes. We considered them as responders (Group A). The remaining 36 patients showed a peak cortisol level below $500 \mathrm{nmol} / \mathrm{L}$; hence, they were considered to be non-responders (Group B). After the execution of ACTH stimulating test, every patient was clinically followed. After the execution of ACTH stimulating test, every patient was clinically followed. Four patients in the Group B were diagnosed for AI (true positive): a female patient with hypopituitarism caused by previous pituitary macroadenoma that had been surgically-removed (secondary AI, baseline cortisol 196 nnmol/L, ACTH 5.3 pg/mL), a male patient with thalassemia, hypogonadism and GH deficiency (secondary AI, baseline cortisol $199 \mathrm{nnmol} / \mathrm{L}, \mathrm{ACTH} 28.8 \mathrm{pg} / \mathrm{mL}$ ), and two female patients with presumed autoimmune adrenalitis (baseline cortisol $28 \mathrm{nmol} / \mathrm{L}$ and $135 \mathrm{nmol} / \mathrm{L}$ and ACTH were $6.4 \mathrm{pg} / \mathrm{mL}$ and $65 \mathrm{pg} / \mathrm{mL}$ ). The last two patients also underwent high-dose $(250 \mu \mathrm{g})$ ACTH test stimulation, and cortisol levels did not reach the cut-off value of $500 \mathrm{nmol} / \mathrm{L}$.

Figure 1 shows serum cortisol values at 0,20 , and 30 minutes after $1 \mu \mathrm{g}$ ACTH administration. A repeated-measures ANOVA with a Greenhouse-Geisser correction showed that cortisol levels were significantly different at 0,20 , and 30 minutes $(F(1520,155.089)=440.41, p<0.01)$. Post-hoc tests using the Bonferroni correction revealed that cortisol levels at zero minutes were statistically significantly different versus cortisol levels after 20 and 30 minutes of $1 \mu \mathrm{g}$ ACTH administration $(p<0.01)$. The test also showed a statistically significant difference between cortisol levels at 20 and 30 minutes $(p<0.01)$. These results showed the importance of the evaluation of cortisol levels at 20 minutes from ACTH administration.

Considering $500 \mathrm{nmol} / \mathrm{L}$ as a cut-off value, low-dose ACTH stimulation showed a 100\% sensitivity and a $67.3 \%$ specificity, enabling the identification of all patients with AI, but with a high rate of FP results. It correctly identified AI only in $11.1 \%$ of patients with positive tests (PPV) and normal adrenal function in $100 \%$ of patients with negative tests (NPV). The test had an accuracy of $68.6 \%$.

Figure 2 shows the ROC curve at 20 and 30 minutes. ROC curve analysis revealed the cut-off of $401.5 \mathrm{nmol} / \mathrm{L}$ to be the best compromise between sensitivity (100\%) and specificity (93.9\%). In this way, the sensitivity remained the same, but the specificity significantly increased (i.e., from $67.3 \%$ to 93.9\%). McNemar's test showed that, by lowering the cut-off value from $500 \mathrm{nmol} / \mathrm{L}$ to $401.5 \mathrm{nmol} / \mathrm{L}$, the number of FPs significantly decreased $(p<0.01)$. In addition, the accuracy of the test significantly increased (i.e., from $68.6 \%$ to $94.1 \%$ ). 


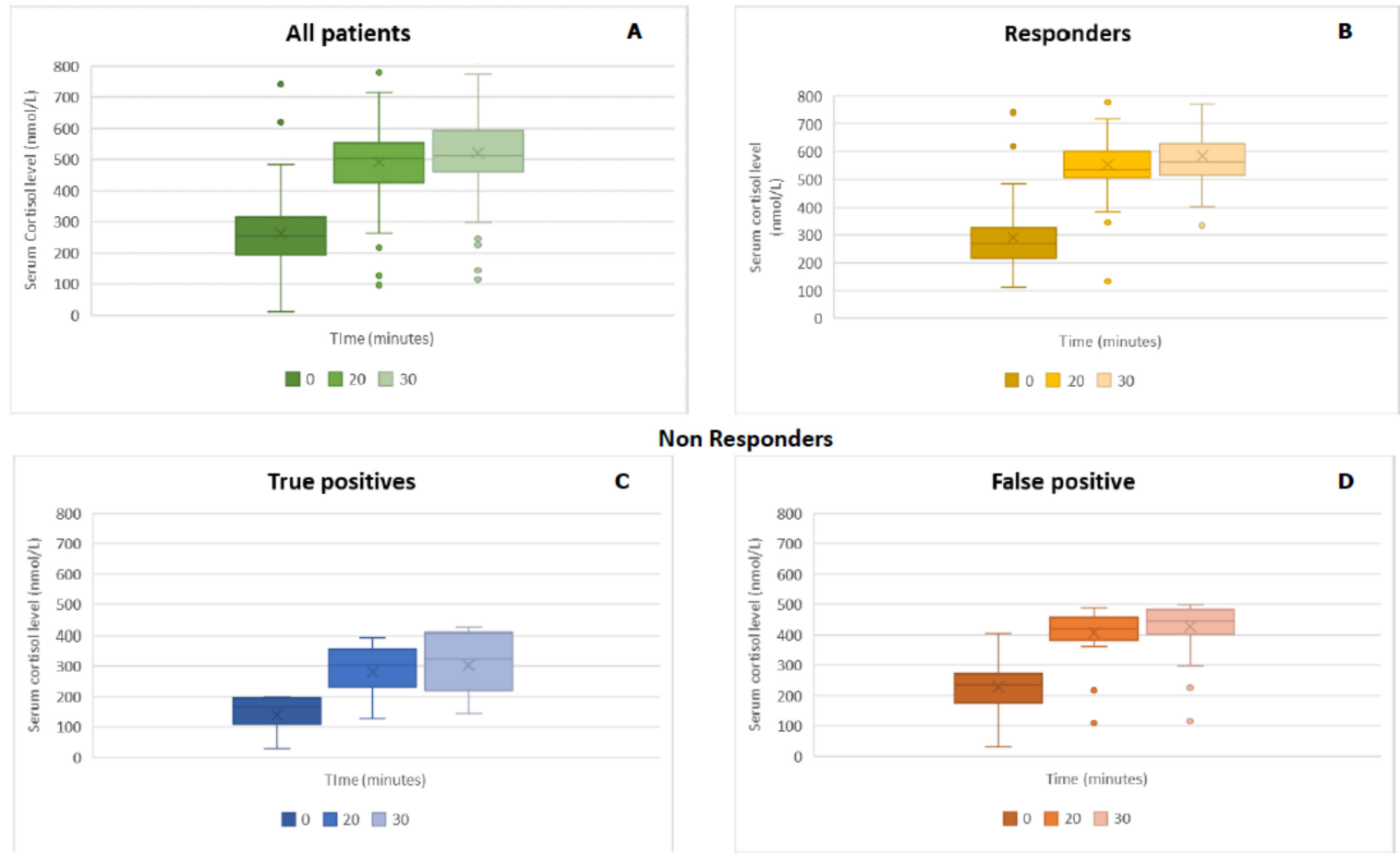

Figure 1. Serum cortisol values at 0,20 , and 30 minutes after corticotropin (ACTH) administration in (A) all patients; (B) the responder group (cortisol peak $>500 \mathrm{nmol} / \mathrm{L}$ ); (C) true positive (cortisol peak $<500 \mathrm{nmol} / \mathrm{L}$, AI diagnosis); and (D) false positive (cortisol peak $<500 \mathrm{nmol} / \mathrm{L}$, but no AI diagnosis).

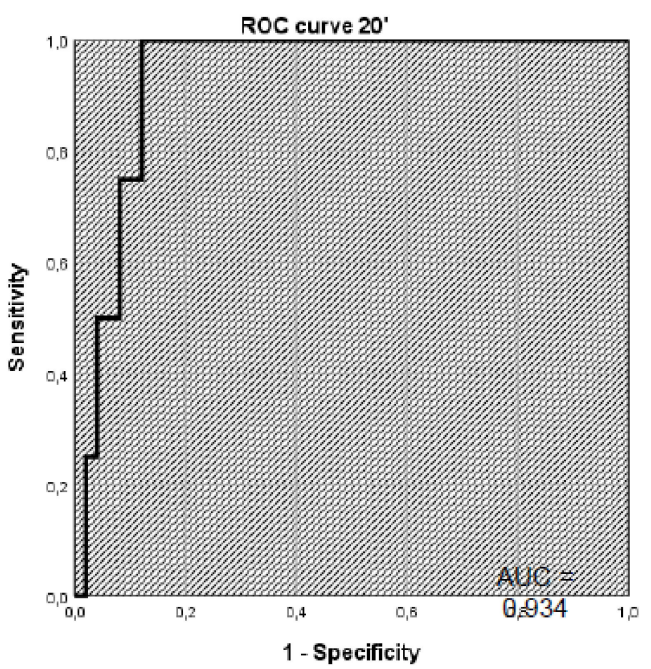

$\mathrm{AUC}=$ area under the curve, $p<0.01$, $95 \%$ confidence interval 0.878-0.991

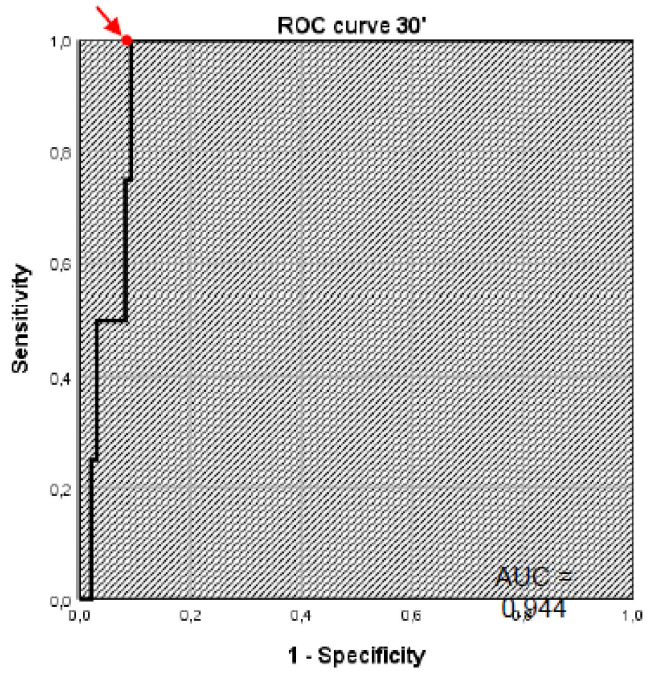

AUC $=$ area under the curve, $p<0.01$, $95 \%$ confidence interval 0.894-0.994

Figure 2. Receiver operating characteristic (ROC) curve of serum cortisol levels at 20 and 30 minutes after ACTH stimulation.

Table 2 summarizes performances of $1 \mu \mathrm{g}$ ACTH stimulation for cut-off levels of $500 \mathrm{nmol} / \mathrm{L}$ and $401.5 \mathrm{nmol} / \mathrm{L}$. 
Table 2. Performances of $1 \mu \mathrm{g}$ ACTH stimulation for cut-off levels of $500 \mathrm{nmol} / \mathrm{L}$ and $401.5 \mathrm{nmol} / \mathrm{L}$.

\begin{tabular}{|c|c|c|}
\hline \multirow{2}{*}{ Parameters } & \multicolumn{2}{|c|}{$1 \mu \mathrm{g}$ ACTH Stimulation Test } \\
\hline & Cut-off of $500 \mathrm{nmol} / \mathrm{L}$ & Cut-off of $401.5 \mathrm{nmol} / \mathrm{L}$ \\
\hline False negative & 0 & 0 \\
\hline False positive & 32 & 3 \\
\hline Sensitivity (\%) & 100 & 100 \\
\hline Specifity (\%) & 67.3 & 93.9 \\
\hline Accuracy $(\%)$ & 68.6 & 94.1 \\
\hline PPV (\%) & 11.1 & 40 \\
\hline NPV (\%) & 100 & 100 \\
\hline
\end{tabular}

\section{Discussion}

A short ACTH stimulation test is actually the "gold standard" for the diagnosis of AI. According to the Endocrine Society guidelines, cortisol levels should be measured after an intravenous administration of ACTH at the standard dose of $250 \mu \mathrm{g}$ for adults and children over two years of age, a dose of $15 \mu \mathrm{g} / \mathrm{kg}$ for newborns, and a dose of $125 \mu \mathrm{g}$ for children under two years, respectively [4], but different protocols are used in clinical practice. Some debates concern the fact that the dosage of $250 \mu \mathrm{g}$ of ACTH is supra-physiological and is capable of generating FN responses in the case of mild forms of AI [3]. Therefore, it has been suggested that a more suitable adrenal evaluation can be obtained, by using a lower dose of ACTH $(1 \mu \mathrm{g})$, and by evaluating cortisol levels at 20 to 30 minutes after ACTH injection [5].

In the present study, cortisol serum levels were evaluated at zero, 20, and 30 minutes after the intravenous injection of $1 \mu \mathrm{g}$ of 1-24 tetracosactid. We found that cortisol levels at zero minutes were significantly different from those measured at 20 and 30 minutes after the administration of $1 \mu \mathrm{g}$ of ACTH. The test also showed also a statistically significant difference between cortisol levels at 20, and 30 minutes, respectively. These results showed the importance of the evaluation of cortisol levels 20 minutes after ACTH administration, since some patients showed peak cortisol levels $>500 \mathrm{nmol} / \mathrm{l}$ at this time-point and $<500 \mathrm{nmol} / \mathrm{l}$ after 30 minutes. Thus, the evaluation only at 30 minutes could be misleading (increasing the positive rate).

To avoid exposure to supraphysiological doses, we chose $1 \mu \mathrm{g}$ ACTH stimulation testing, since studies in the literature have shown its reproducibility [5] and comparable results with the high-dose test [4]. In 2015, Ospina et al. published a systematic review and meta-analysis about the diagnostic accuracy of the high $(250 \mu \mathrm{g})$ and $1 \mu \mathrm{g}$ (i.e., low-dose) ACTH stimulation test in the diagnosis of AI, both central and primary. Both tests showed similar diagnostic accuracy (low sensitivity and high specificity) in the diagnosis of central AI [6]. Regarding primary forms, the data available for the $1 \mu \mathrm{g}$ test were insufficient to draw conclusions. However, the use of $1 \mu \mathrm{g}$ testing could be useful for the early diagnosis of the initial or partial stages of primary AI [7].

Regarding the timing of blood sampling for cortisol evaluation, it has been reported that, the lower the dose of ACTH is, the earlier the timing of the peak cortisol levels is [8] and, for the $1 \mu \mathrm{g}$ ACTH stimulation test, the peak cortisol levels occurred after about 20 minutes [9].

Previous studies have shown that cortisol values of $497 \mathrm{nmol} / \mathrm{L}$ and above, in healthy subjects undergoing $1 \mu \mathrm{g}$ ACTH stimulation, are appropriate, and many authors have previously used this cut-off value [10-12]. We considered $500 \mathrm{nmol} / \mathrm{L}$ as a cut-off value, which was used to distinguish responders from non-responders. We found that $1 \mu \mathrm{g}$ ACTH stimulation testing presented a $100 \%$ sensitivity, a $67.3 \%$ specificity, and a $68.6 \%$ accuracy, allowing us to identify patients with AI, but with a high rate of FP results. We also found that, by reducing the cut-off to $401.5 \mathrm{nmol} / \mathrm{L}$, the test sensitivity did not change, whereas the specificity significantly increased and the number of FPs significantly 
decreased. Moreover, after the ACTH stimulation test, every patient was clinically followed and only four patients $(3.9 \%)$ were diagnosed for AI (true positive). This finding is in agreement with epidemiological data, which indicates a prevalence of about 100 to 140 cases per million and an incidence of 4:1,000,000 per year in Western societies [4].

To our knowledge, this is the first study that evaluated sensitivity and specificity of $1 \mu \mathrm{g}$ ACTH stimulation testing by using a lower cut-off.

Although peak cortisol levels below $500 \mathrm{nmol} / \mathrm{L}(18 \mu \mathrm{g} / \mathrm{dL})$ are usually suggestive of AI, the normal cortisol response to ACTH testing remains not completely defined, possibly due to the various subject- and test-condition-related factors, such as the use of oral contraception, the degree of fat mass, and the methods of assay [13]. Some authors evaluated the response to ACTH stimulation testing using higher cut-off values and found several subnormal responses, resulting in further increased FP responses [5]. Tordjman et al. performed ROC analysis to evaluate the following different potential cut-off values after 1- $\mu \mathrm{g}$ ACTH stimulation: 275, 415, 470, 500, 550, 605, and $690 \mathrm{nmol} / \mathrm{L}$. The authors found that, by using a stimulated cortisol level of above $500 \mathrm{nmol} / \mathrm{L}$, the test had $94.7 \%$ sensitivity, $90 \%$ specificity, and $91 \%$ accuracy [14]. We showed that, by using $401.5 \mathrm{nmol} / \mathrm{L}$ as a cut-off value, test sensitivity was $100 \%$, test specificity was $93.9 \%$, and test accuracy was $94.1 \%$. Hence, this value of cortisol improved all parameters in comparison with the other studies.

\section{Conclusions}

Low-dose $(1 \mu \mathrm{g})$ ACTH testing is useful for AI diagnosis, by avoiding exposure on the part of the patient to a supraphysiological ACTH dosage. Although cortisol values of less than $500 \mathrm{nmol} / \mathrm{L}$ are usually considered suggestive of AI, the correct cortisol cut-off to use remains under debate. We showed that, by using a cut-off value of $401.5 \mathrm{nmol} / \mathrm{L}$ for low-dose ACTH stimulation, the number of FPs could be significantly diminished, maintaining an elevated sensitivity and thus improving the accuracy. Further studies with a greater number of patients are useful to confirm these findings.

\section{Limitation}

The study lacks a comparison with the $250 \mu \mathrm{g}$ ACTH stimulation test. Although there are many debates on this diagnostic test, today it has to be considered as the gold standard for the diagnosis of AI. Further studies will be needed.

Author Contributions: Conceptualization, investigation, methodology, writing-original draft, L.M.M.; visualization, R.A.C.; data curation, formal analysis, investigation, F.B.; visualization, R.C. and S.L.V.; project administration, supervision, validation, A.E.C.

Conflicts of Interest: The authors declare no conflict of interest.

\section{References}

1. Bensing, S.; Hulting, A.L.; Husebye, E.S.; KämpeO Løvås, K. Management of endocrine disease: Epidemiology, quality of life and complications of primary adrenal insufficiency: A review. Eur. J. Endocrinol. 2016, 175, R107-R116. [CrossRef] [PubMed]

2. Arlt, W.; Allolio, B. Adrenal insufficiency. Lancet 2003, 361, 1881-1893. [CrossRef]

3. Chatha, K.K.; Middle, J.G.; Kilpatrick, E.S. National UK audit of the short synacthen test. Ann. Clin. Biochem. 2010, 47, 158-164. [CrossRef] [PubMed]

4. Bornstein, S.R.; Allolio, B.; Arlt, W.; Barthel, A.; Don-Wauchope, A.; Hammer, G.D.; Husebye, E.S.; Merke, D.P.; Murad, M.H.; Stratakis, C.A.; et al. Diagnosis and treatment of primary adrenal insufficiency: An endocrine society clinical practice guideline. J. Clin. Endocrinol. Metab. 2016, 101, 364-389. [CrossRef] [PubMed]

5. Park, Y.J.; Park, K.S.; Kim, J.H.; Shin, C.S.; Kim, S.Y.; Lee, H.K. Reproducibility of the cortisol response to stimulation with the low dose (1 microg) of ACTH. Clin. Endocrinol. (Oxf) 1999, 51, 153-158. [CrossRef] [PubMed] 
6. Ospina, N.S.; Al Nofal, A.; Bancos, I.; Javed, A.; Benkhadra, K.; Kapoor, E.; Lteif, A.N.; Natt, N.; Murad, M.H. ACTH Stimulation tests for the diagnosis of adrenal insufficiency: Systematic review and meta-analysis. J. Clin. Endocrinol. Metab. 2016, 101, 427-434. [CrossRef] [PubMed]

7. Pura, M.; Kreze, A. Jr.; Kentos, P.; Vanuga, P. The low-dose (1 microg) cosyntropin test (LDT) for primary adrenocortical insufficiency: Defining the normal cortisol response and report on first patients with Addison disease confirmed with LDT. Exp. Clin. Endocrinol. Diabetes 2010, 118, 151-157. [CrossRef] [PubMed]

8. Daidoh, H.; Morita, H.; Mune, T.; Murayama, M.; Hanafusa, J.; Ni, H.; Shibata, H.; Yasuda, K. Responses of plasma adrenocortical steroids to low dose ACTH in normal subjects. Clin. Endocrinol. (Oxf) 1995, 43, 311-315. [CrossRef] [PubMed]

9. Rasmuson, S.; Olsson, T.; Hagg, E. A low dose ACTH test to assess the function of the hypothalamic-pituitary-adrenal axis. Clin. Endocrinol. (Oxf.) 1996, 44, 151-156. [CrossRef] [PubMed]

10. Broide, J.; Soferman, R.; Kivity, S.; Golander, A.; Dickstein, G.; Spirer, Z.; Weisman, Y. Low-dose adrenocorticotropin test reveals impaired adrenal function in patients taking inhaled corticosteroids. J. Clin. Endocrinol. Metab. 1995, 80, 1243-1246. [PubMed]

11. Schultz, C.H.; Rivers, E.P.; Feldkamp, C.S.; Goad, E.G.; Smithline, H.A.; Martin, G.B.; Fath, J.J.; Wortsman, J.; Nowak, R.M. A characterization of hypothalamic-pituitary-adrenal axis function during and after human cardiac arrest. Crit. Care Med. 1993, 21, 1339-1347. [CrossRef] [PubMed]

12. Tordjman, K.; Jaffe, A.; Grazas, N.; Apter, C.; Stern, N. The role of the low dose (1 microgram) adrenocorticotropin test in the evaluation of patients with pituitary diseases. J. Clin. Endocrinol. Metab. 1995, 80, 1301-1305. [PubMed]

13. Klose, M.; Lange, M.; Rasmussen, A.K.; Skakkebaek, N.E.; Hilsted, L.; Haug, E.; Andersen, M.; Feldt-Rasmussen, U. Factors influencing the adrenocorticotropin test: Role of contemporary cortisol assays, body composition, and oral contraceptive agents. J. Clin. Endocrinol. Metab. 2007, 92, 1326-1333. [CrossRef] [PubMed]

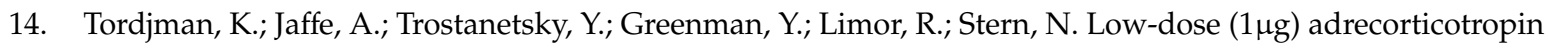
stimulation as a screening test for impaired hypothalamo-pituitary-adrenal axis function: Sensitivity, specificity and accuracy in comparison with the high-dose $(250 \mu \mathrm{g})$ test. Clin. Endocrinol. (Oxf.) 2000, 52, 633-640. [CrossRef] [PubMed] 